\title{
Progression of Jackhammer Esophagus to Achalasia: Author's Reply
}

TO THE EDITOR: We would like to thank Huang and Rezaie for their interesting comments. As the authors are well aware of, diagnosis of Jackhammer esophagus requires normal integrated residual pressure (IRP) and at least $20 \%$ of the swallows with distal contractile integral (DCI) that is greater than $8000 \mathrm{mmHg} \cdot \mathrm{sec} \cdot \mathrm{cm}$, using the Chicago classification version $3{ }^{2}$ In our patient, only $30 \%$ of the swallows were hypercontractile and the median IRP was within the normal range $(7 \mathrm{mmHg})$. When each swallow was reviewed, a few of the measured IRP's were above the normal range (18-20 mmHg), but the median IRP remained within the normal range.

The authors suggested in their letter that patients with Jackhammer esophagus who progress to achalasia tend to have abnormally high median IRP. In addition, the authors pointed out to their recent publication, demonstrating that patients with Jackhammer esophagus and elevated IRP progressed within 2 years to type 3 achalasia. ${ }^{3}$ However, one has to recall that based on the latest Chicago classification, patients with abnormally high IRP and esophageal hypercontractility following more than $20 \%$ of the swallows do not fall under the category of Jackhammer esophagus. In fact, these patients demonstrate esophagogastric junction outflow obstruction with hypercontractile swallows, likely in response to the obstruction at the esophagogastric junction. Those type of patients have already been recognized in the literature to potentially represent early achalasia. $^{4}$

Our case do raise an interesting question, is Jackhammer esophagus a unique and durable esophageal motor disorder? As Huang and Rezaie ${ }^{1}$ pointed out in their letter, at the time the Jackhammer esophagus was diagnosed, the patient demonstrated on barium swallow, mildly dilated esophagus and narrowed gastroesophageal junction. ${ }^{5}$ These findings may suggest early achalasia, but the high resolution esophageal manometry revealed Jackhammer esophagus. In addition, it is possible that Jackhammer esophagus may not represent a unique esophageal motor disorder, but rather an esophageal response in the background of esophageal motor function abnormality. Thus, the presence of swallows induced hypercontractility is a response to an underlying esophageal motor disorder, such as outflow obstruction, rather than a unique and durable esophageal motor disorder. Lastly, we would like to reassure Huang and Rezaie ${ }^{1}$ that we re-evaluated the values of the recorded IRP's in our patient and the median IRP remains within the normal range as we originally reported.

Jason Abdallah and Ronnie Fass The Esophageal and Swallowing Center, Division of Gastroenterology and Hepatology, MetroHealth Medical Center, Case Western Reserve University School of Medicine Cleveland, Ohio, USA

1. Huang L, Rezaie A. Progression of Jackhammer esophagus to achalasia. J Neurogastroenterol Motil 2016;22:348-349.

2. Kahrilas PJ, Bredenoord AJ, Fox M et al. The Chicago Classification of esophageal motility disorders, v3.0. Neurogastroenteral Motil 2015:27:160-174.

3. Huang L, Rezaie A, Busseri B, Change C, Pimental M. Natural manometric course of Jackhammer esophagus and its determinants; a largescale database analysis. Gastroenterology 2014;146(suppl 1):S679.

4. Schere JR, Kwiatek MA, Soper NJ, Pandolfino JE, Kahrilas PJ. Functional esophagogastric junction obstruction with intact peristalsis a heterogeneous syndrome sometime akin to achalasia. J Gastrointest Surg 2016;13:2219-2225

5. Abdallah J, Fass R. Progression of Jackhammer esophagus to type 2 achalasia. J Neurogastroenteral Motil 2016;22:153-156.

\section{Conflicts of interest: None}

